\title{
Overview of segmented glass optics development for the Constellation-X hard X-ray telescope
}

Charles J. Hailey, Finn Erland Christensen, William W. Craig, Fiona A. Harrison, Jason Koglin, et al.

Charles J. Hailey, Finn Erland Christensen, William W. Craig, Fiona A. Harrison, Jason Koglin, Robert Petre, David L. Windt, William W. Zhang, "Overview of segmented glass optics development for the Constellation-X hard X-ray telescope," Proc. SPIE 4851, X-Ray and Gamma-Ray Telescopes and Instruments for Astronomy, (11 March 2003); doi: 10.1117/12.461487

Event: Astronomical Telescopes and Instrumentation, 2002, Waikoloa, Hawai'i, United States 


\title{
Overview of Segmented Glass Optics Development for the Constellation-X Hard X-ray Telescope
}

\author{
C.J. Hailey ${ }^{a}$, F.E. Christensen ${ }^{\text {b }}$ W.W. Craig ${ }^{\text {, }}$ F.A. Harrison ${ }^{\text {d }}$, J. Koglin ${ }^{\mathrm{a}}$, \\ R. Petre ${ }^{\mathrm{e}}$, D.W. Windt ${ }^{\mathrm{a}}$, W. Zhang ${ }^{\mathrm{e}}$
}

\begin{abstract}
We report recent work on segmented glass optics for the Constellation-X Hard X-ray Telescope. This effort seeks to both improve the figure of the free-standing glass substrates, and to refine a newly-developed mounting technology for the substrates. We discuss metrology on recently characterized glass shells both unmounted and mounted. We also present plans for several prototype optics to be constructed in the upcoming year.
\end{abstract}

Keywords: Constellation-X, X-ray optics, Hard X-ray telescope

\section{INTRODUCTION}

The Constellation-X mission will carry 12 hard X-ray telescopes (HXT), three on each of four coaligned satellites. These telescopes are designed to complement the soft X-ray telescopes (SXT), matching their spectroscopic sensitivity for continuum observations in the energy band from $6 \mathrm{keV}$ up to at least $40 \mathrm{keV}$. Although the HXT has a modest angular resolution requirement (60") and goal (30") compared to the SXT requirement (15"), the tight constraints on cost and weight ( $\leq 250 \mathrm{~kg}$ total mass excluding structure), combined with the large required geometric area $(\geq 1500$ $\mathrm{cm}^{2}$ ) at higher energies, provide a number of technical challenges. The relevant optics design parameters are in table 1.

\begin{tabular}{|l|l|}
\hline Focal length & $10 \mathrm{~m}$ \\
\hline Minimum shell radius & $3 \mathrm{~cm}$ \\
\hline Maximum shell radius & $20 \mathrm{~cm}$ \\
\hline Shell length & $25 \mathrm{~cm}$ \\
\hline Shell thickness & $300 \mu \mathrm{m}$ \\
\hline Number of shells per telescope & 149 \\
\hline Telescope mass & $190 \mathrm{~kg}$ \\
\hline
\end{tabular}

Table 1. Optics parameters for the Constellation-X Hard X-ray Telescopes.

Two approaches for the HXT are currently under development; Nickel replica optics, in which the shells are monolithic ${ }^{1}$ and segmented glass optics, which we are pursuing. The glass optics use the conic approximation to the Wolter geometry. For the HXT optical design, the degradation in performance due to the conic approximation is negligible. In fact, the telescope fabrication approach described below could produce an exact Wolter geometry. The telescope layers are built out of small, individual glass segments. The discussion employs the following terms. A shell is a complete figure of revolution containing an upper layer and lower layer (the parabola and hyperbola of an exact Wolter I geometry). The glass pieces used to construct the layers are called segments.

${ }^{a}$ Contact author: chuckh@astro.columbia.edu Columbia Astrophysics Laboratory, 550 W. 120 St., New York, NY 10027; ${ }^{b}$ Danish Space Research Institute, Juliane Maries Vej 30,Copenhagen Dk-2100, Denmark; 'Lawrence Livermore National Laboratory, 8000 East Ave., Livermore, CA 94550; ${ }^{\mathrm{d} C a l T e c h}$ 220-47, Pasadena, CA 91125; ${ }^{\mathrm{N}}$ ASA/Goddard Space Flight Center, Greenbelt, MD 20771 
The HXT will require glass $\sim 0.2-0.3 \mathrm{~mm}$ thick in order to meet the mass goal for Constellation-X $(0.6 \mathrm{~mm}$ glass could meet the requirement, however reducing mass to the target level is an important goal). The glass segments will be built up into shells. The number of glass segments in a single shell is a design parameter. As described below, the individual layers have been built out of 1-4 axial segments and 4-8 azimuthal segments. Therefore individual shells would have 832 glass segments. For comparison the SXT is proposed to have 12 azimuthal segments.

The segmented glass optics development naturally falls into three distinct areas; glass segment production; optics assembly; metrology and testing. We discuss each of these areas below as well as overall plans for construction and testing of the prototype HXT.

\section{MIRROR FABRICATION APPROACH}

The Constellation-X prototype mirrors will use epoxy-replicated glass (hereafter called eGlass). The eGlass mirror segments are fabricated in a three-step process. First, a flat glass sheet is thermally-slumped onto a convex forming mandrel to create a substrate. The microsheet glass is primarily used in the flat panel display industry and is notable for its atomic smoothness. The edges on all four sides of the glass are trimmed because they are typically poorly formed and do not conform to the forming mandrel as desired. Although the substrate has an overall figure that is very close (better than $1 \mu \mathrm{m}$ everywhere) to that of the forming mandrel, it has many mid-frequency ripples which probably come from two sources: the original glass surface itself and the slumping process which causes the outside of the substrate to stretch and the inside to buckle. Second, the inside of this substrate is coated with a layer $(\sim 10 \mu \mathrm{m})$ of epoxy. Third, this epoxycoated substrate is brought together in a vacuum chamber with a replication mandrel which has very good mid-frequency figure. The replication mandrel is coated with the desired multilayers. After the epoxy is cured at a slightly elevated temperature, the substrate with the attached multilayer is peeled off the replication mandrel. This is the same production process as the SXT mirror segments except for the multilayer-coated replication mandrel.

A typical result for an early eGlass mirror, obtained with an interferometer, is displayed in figure 1. Three axial scans are shown along a $10 \mathrm{~cm}$ mirror and they illustrate the suppression of mid-frequency ripple. The mirror performance, if perfectly mounted, would be 10" (HPD). This is comparable to the error introduced in mounting (below). More details of the error budget breakdown for the mirror are presented elsewhere in the Proceedings ${ }^{3}$. Mounted performance will be determined with the prototype described below.

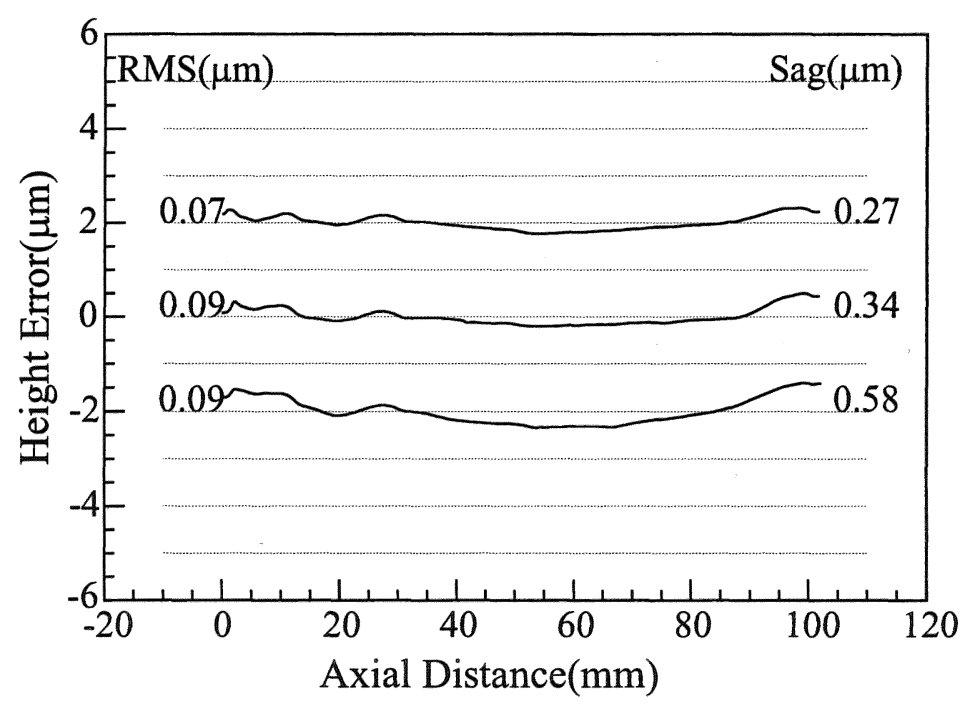

Figure 1: Axial scans of eGlass demonstrating surface figure exceeding the HXT goal. 


\section{ASSEMBLY MACHINE AND OVERCONSTRAINED MOUNTING APPROACH}

All current work on the overconstrained mounting approach and assembly machine has been done at Colorado Precision Products Inc., in Boulder Co.. CPPI is experienced in space-qualified fabrication, having machined the $\mathrm{SiC}$ grating substrates for the Reflection Grating Spectrometer on XMM-Newton. CPPI is also building the HEFT telescopes.

Assembly of the glass segments into telescopes follows closely the approach developed for the HEFT optics ${ }^{4,5}$. A cartoon of this process is shown in figure 2. Fabrication begins with a lightweight, hollow mandrel, such as Titanium. The telescope mandrel is mounted on a spindle with arcsecond rotation accuracy. Graphite rods of length comparable to the mandrel are epoxied axially along it, as well as azimuthally around the mandrel. Once the graphite rods are epoxied to the glass, the rods are machined. Moore translation stages are used for positioning the cutting tool with respect to the graphite rods. The surfaces of the rods precisely match the radius at which the front surface of the glass segment must sit. Thus the front surface of the glass is guaranteed to be at the correct position for X-ray reflection. The proper radius of the graphite rods is determined

through the use of radial gauges, which are positioned above the optic on a kinematic mounting stage. The alignment of the mandrel to the spindle axis is not crucial since the spindle axis is used to define the optic axis, and the spacers are machined with respect to the spindle axis. A thin bead of epoxy is applied to the rods with a pressurized hand applicator. The bondline thickness is small $(\sim 10 \mu \mathrm{m})$ and the viscosity of the epoxy is chosen to avoid excessive runout.. The upper and lower layers of glass are laid down on the rods and fastened in position using metal shoes that run the length of the upper and lower layers respectively. The shoes are locked into position along "wagon wheels"
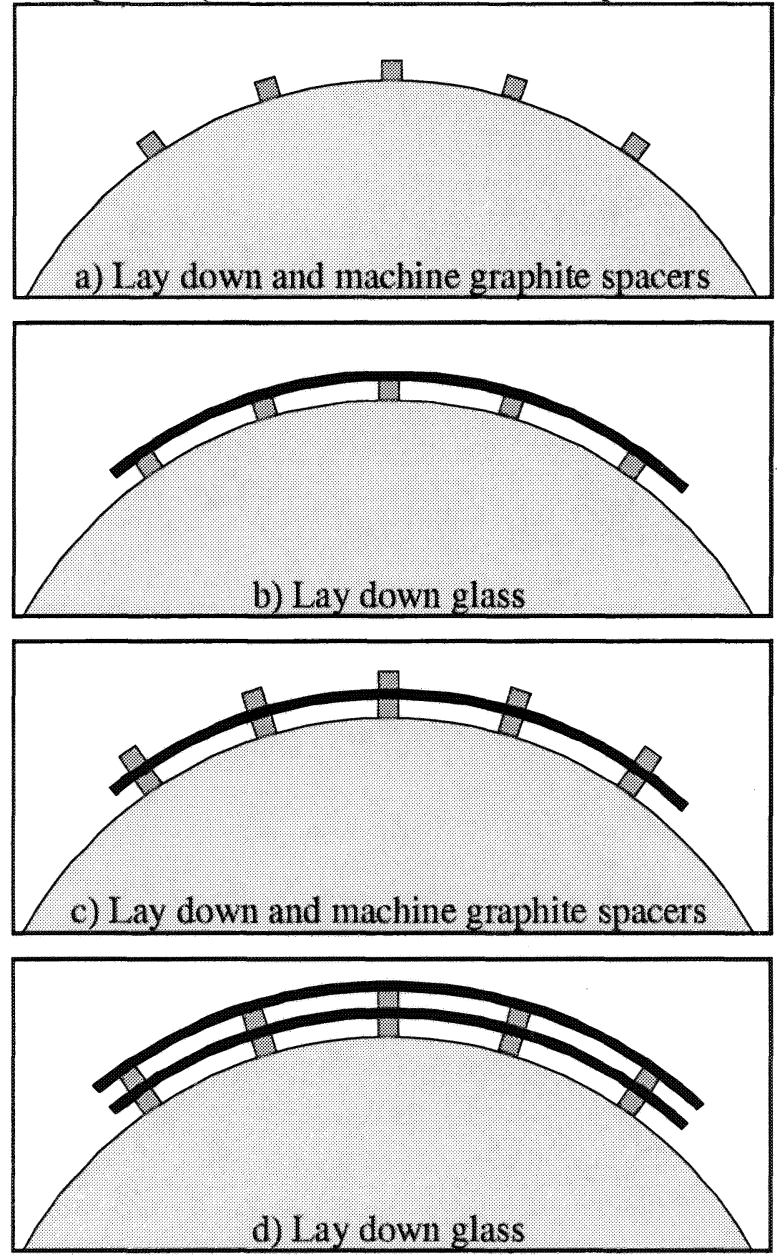

Figure 2: Telescope Assembly. A glass-graphite rod-epoxy matrix is built up from a telescope mandrel. The rods for each shell are machined so that the front surface of the glass is at the proper position, leading to zero stackup errors. 
(figure 5) located at the end of the telescope mandrel. The shoes provide pressure on the glass to ensure good bonding of the glass to the rods. The spindle is rotated and then next set of glass layers is laid down. The process is repeated until all the sectors making up the shell are in place. Once the epoxy has cured another layer of graphite rods will be applied on top of the glass and the process repeated. The graphite rods serve two purposes. They serve as spacers to define the gap between the shells. Hence the terms spacer and rods are used interchangeably in this paper. The rods also force the glass to comply to the conic shape of the mandrel. A dust removal system is used during the machining operations to prevent particulates from accumulating on the rods and optical surfaces during epoxying and subsequent metrology.

This assembly scheme has some notable features. Firstly there is a complete integration of the assembly and alignment steps. This is a divergence from previous approaches to segmented optics assembly, where the segments were first mounted and then aligned either individually or as a small group of reflectors ${ }^{6,7}$. Secondly there are no stackup errors in this mounting approach; the spacers of each successive shell are machined to the precise radial location at which front surface of the next glass shell will be mounted.

\subsection{Investigations of Telescope Fabrication Approach}

Preliminary investigation has been performed on the approach to HXT telescope construction described here. The ultimate telescope performance depends on the figure of the free-standing glass segments and on the errors contributed from the assembly machine. In principle the graphite rod machining defines both the optical axis of the telescopes and the exact positions which should be assumed by the glass reflecting surfaces. In practice errors in positioning these spacers in translation and angle, figure errors in machining the spacers and errors in defining the optical axis will all lead to distortions of glass segments even if they have "zero" figure error. Additional complications arise because the mounting process itself can introduce distortions into the glass unless particular care is taken with issues such as epoxy uniformity and uniform application of loads during glass compression during the curing process. Ultimate limits on performance will also depend on a complicated interplay of the overconstraint with the residual free-standing figure errors of the glass (simple and higher order bows and twists) which exist even after replication of the glass. In practice (see below) we do not expect this to be a problem until we seek performance well beyond that of the HXT goal.

\subsubsection{Assembly Machine Characterization}

Figure 3 shows the assembly machine with the kinematic metrology frame containing the radial probes. Details of the assembly machine and its characterization are presented elsewhere ${ }^{8}$ so we make only some general comments on performance. The most relevant point for the HXT is that the assembly machine has already demonstrated performance such that it will not contribute significantly to the overall HXT angular resolution error

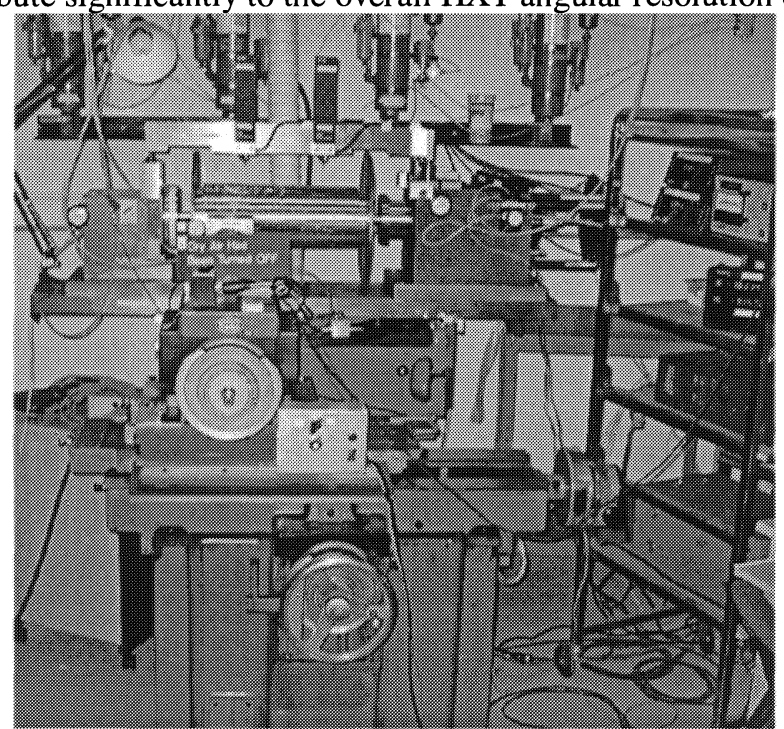

Figure 3: The assembly machine. The radial probes and metrology frame (top), optics (center) and metrology station (right). 
budget. This has been demonstrated using Linear Voltage Differential Transformer (LVDT) metrology. The LVDT probe can scan the length of the spacers and provide good relative and absolute surface metrology. The LVDT probe, developed at CPPI, provides only $0.1 \mathrm{gm}$ of force on the spacers, not enough to distort the measurements. Moreover the LVDT uses an air bearing and constant air pressure to maintain a constant force during surface measurement. This leads to greater measurement accuracy. In addition the probe motion is extremely stiff in the radial direction so that it is essentially a zero force metrology tool. Figure 4 shows a scan of some spacers laid side by side.

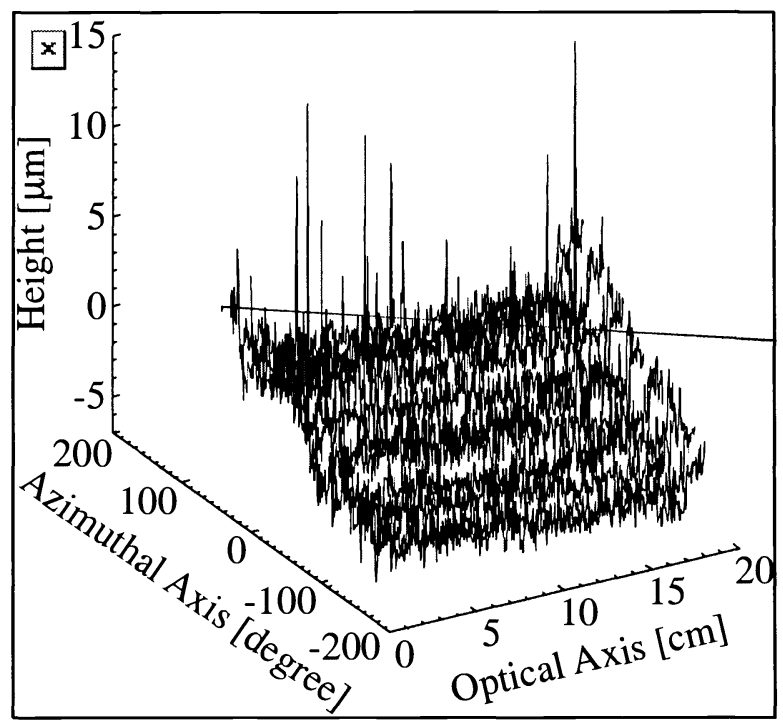

Figure 4: Axial LVDT measurements are plotted with respect to their axial position on the optic for an entire complement of machined spacers. The machined spacers have a high frequency peak to valley roughness of about $1.5 \mu \mathrm{m}$.

The RMS amplitude of the spacer figure is typically $1.5 \mu \mathrm{m}$. There is some high frequency roughness associated with the spacer machining. However this roughness does not in any way compromise the performance of the mounted glass because the glass cannot be deformed on the typical length scale of the roughness. Indeed the small roughness associated with the spacer increases the strength of the epoxy bondline. The uniformity of the epoxy bondline is important even in the case of "perfect" glass. Separate tests of the glass thickness combined with metrology on the glass along the point of glass-spacer contact allows us to estimate $\sim 1-2$ " contribution of the bondline to the assembly error budget. This is very good and attributable to the small thickness of epoxy $(\sim 10 \mu \mathrm{m})$ obtained with a low viscosity epoxy. For comparison, the dominant contribution to the assembly machine error budget is the figure error in the machined spacers, which is $\sim 7$ ". The overall error contribution from the assembly machine is $~ 8-9$ ". This is a small contribution relative to the HXT goal of 30 " and smaller than even the error introduced by the conic approximation to the Wolter I.

\subsection{Prototype Telescope Results}

We have evaluated the overconstrained mounting process and the assembly machine through construction of a series of prototype telescopes. A typical prototype is shown in figure 5 where the graphite rods are clearly visible along the length of the mandrel. One major difference between these prototype telescopes and our proposed Constellation-X HXT prototoype telescope is in the glass segments. The prototypes used the original approach developed for thermallyslumped glass X-ray optics, which involves slumping into a concave mandrel without epoxy replication ${ }^{9}$ (called tGlass below). The motivation for this approach was to separately investigate the ultimate performance that could be obtained with tGlass. This is also the approach that is followed for the HEFT telescopes, where the goal is a more modest 60", achievable with tGlass rather than eGlass. Performance better than 60" can be obtained with tGlass. This requires cutting down the glass into shorter segments both axially and azimuthally. Consequently the tGlass will asymptotically approach the performance of the eGlass in the limit of smaller glass segments. Even this is not precisely true since the 
intrinsic stiffness of the tGlass is relevant and depends on the segment size. However the simplest interpretation of the results described below is that even tGlass obtains performance very near the Constellation-X HXT goal for the outer radii and that the results on the prototypes are completely limited by tGlass figure error, not the assembly machine error. Cutting down to short segments will not be required with eGlass because of its good figure.

The prototype results of significance to the HXT were primarily obtained with a design utilizing octant glass (glass segments consisting of 35 deg sectors). The upper and lower layers were $20 \mathrm{~cm}$ long and each layer had $45 \mathrm{~cm}$ segments. The focal length used was $6.5 \mathrm{~m}$ and the diameter of the pieces was $\sim 18 \mathrm{~cm}$ (comparable to the mid-radius range of HXT shells). In one case glass was utilized which was $0.2 \mathrm{~mm}$ thick. This prototype

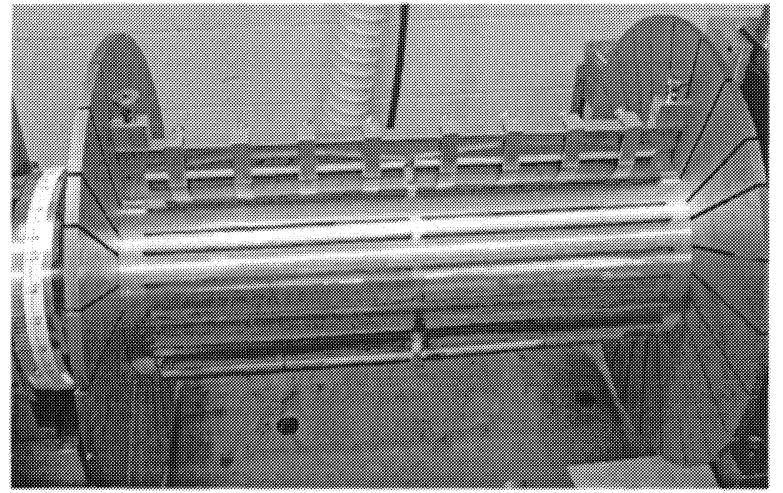

Figure 5: Prototype HXT optic. The upper/lower glass layers and spacers are visible. The metal bar with U-shaped clamps is a glass carrier for mounting the upper and lower glass segments. The wagon wheels are visible at the ends of the telescope mandrel.

obtained performance of 51" (HPD), and is the first instance in which a prototype has been demonstrated which exceeds the HXT performance requirement while simultaneously meeting the mass goal. The performance of this prototype was completely limited by the figure error in the free-standing tGlass. We have also done very preliminary work on mounting even thinner glass with the assembly machine $(\sim 100 \mu \mathrm{m}$ and $145 \mu \mathrm{m})$. The former glass is too thin to work with comfortably and the breakage rate would likely make it prohibitively difficult to use with the assembly machine. We have mounted a few segments of $145 \mu \mathrm{m}$ glass with performance $\sim 60$ ", again limited by the figure of the glass. The most significant results for a prototype (figure 6) were obtained using $0.3 \mathrm{~mm}$ glass with the other specifications of the glass and optics as described above. This prototype obtained 40" (HPD) as determined from X-ray pencil beam scans combined to form a full image ${ }^{10}$. A separate determination of the telescope performance can be obtained by combining LVDT scans of the back surfaces of the glass segments as they are being laid down. These LVDT scans are combined with a ray trace

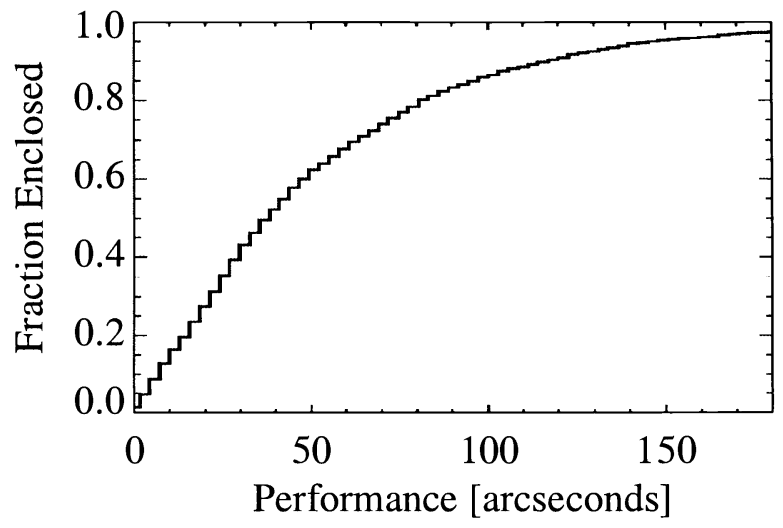

Figure 6: LVDT scans of a prototype telescope constructed with mounting technique described here. The overall performance was $\sim 40$ "(HPD), near the Constellation-X goal. 
code to estimate telescope performance. This approach relies on the fact that for the tGlass the thickness variation from front to back surface is not large enough ( $\sim$ few arcseconds) to contribute a significant uncertainty to the resolution determination.

The primary difference between the $0.2 \mathrm{~mm}$ thick glass results and the $0.3 \mathrm{~mm}$ glass is that the figure was better on the $0.3 \mathrm{~mm}$ glass segments. However the $0.3 \mathrm{~mm}$ glass would have performed even better had it been better radially matched to the telescope mandrel. This can be seen by observing the LVDT scans of the mounted glass (figure 7). There is significant in-phase roundness error due to this radial mismatch. Based on the free-standing figure of the glass used in this telescope, we predict the performance would have been $~ 25$ " (HPD) rather than $~ 40$ "(HPD) without the radial mismatch. The error at that point would have been dominated by a residual out of phase roundness error present in the glass, rather than due to the ( $\left.\sim 8^{\prime \prime}\right)$ contribution from the mounting process. These results with tGlass demonstrate the capability of the assembly approach to meet the HXT performance goal. Even better results would be obtained with eGlass, whose surface figure would not be the limiting factor in angular resolution at all.

\subsection{High Throughput Production Issues}

From the standpoint of high throughput telescope production the mounting approach is also very attractive. On HEFT we produce one shell per day using one assembly machine. Throughput can be increased in many ways. The lengthiest parts of the assembly process are the machining of the spacers and the curing of the epoxy. A high degree of parallelism is possible. A telescope can be removed from the spindle after a shell of glass is laid and while the epoxy is curing. A second telescope can then be mounted on the spindle for spacer machining. We have already verified that we can remount a HEFT telescope on the

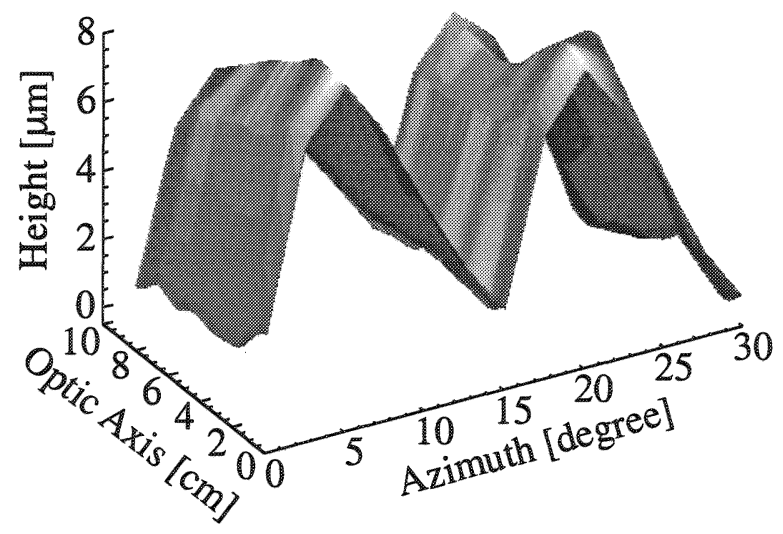

Figure 7: In-phase roundness error due to radial mismatch of glass and mandrel in tGlass prototype telescope.

machine for further assembly. In fact this is a necessity when we want to perform $R \& D$ on separate prototype telescopes. Therefore 2 shells can be laid per day per machine and multiple machines can be operated in parallel. Concentric multimandrel designs can be used to reduce risk during the production process and improve production efficiency by dedicating separate machines to building different radial segments of the telescope. For instance, a three mandrel design, with a dedicated assembly machine for each mandrel, permits laying $\sim 18$ shells per week ( 180 segments per week in a quintent design). While this calculation is merely illustrative, it shows that high production rates can be sustained with this mounting approach and only a few assembly machines. The numbers would increase with more machines.

While the exact HXT geometry for a segmented glass telescope is not yet defined, the number of glass segments per shell pose no obstacles to production regardless of the design chosen. The actual time required to epoxy the spacers, lay down the glass segments and immobilize the glass segments for curing with epoxy is a small part of the assembly time budget. This is a consequence of our ability to handle each upper and lower glass layer as a monolithic glass section. 
This is illustrated in figure 5 where a glass carrier developed for this purpose is shown. The ease of handling multiple glass segments during the assembly process will allow us considerable optics design flexibility. The mirror production throughput is not a problem because it is highly amenable to parallel implementation. Of order 50-100 mirror segments can be fabricated per day. The bottle-neck of segment production is deposition of the multilayer coatings.

\subsection{Environmental Testing}

A prototype telescope with 24 shells has already been environmentally tested to establish the flight-worthiness of telescopes produced with the approach described here. This prototype was tested in all three axes to the Delta IV qualification level vibration spectrum with no apparent degradation of the glass. The lowest natural frequency of the glass between supports is approximately $700 \mathrm{~Hz}$. This high natural frequency will not couple with the low frequency spectrum typically found on rockets.

\section{PROTOTYPE PERFORMANCE SUMMARY AND FUTURE PLANS}

Research has demonstrated both glass segments and a mounting concept which together should meet or exceed the HXT performance goals. The next step is to construct a prototype telescope which combines the eGlass with the overconstrained mounting approach. The design of the prototype telescope is currently under study but will likely consist of quadrant or quintant sectors. The high quality of the eGlass suggests that each layer will consist of only one glass segment. The glass will be characterized with interferometry and laser reflectometry. This data will be used with extant ray trace codes and a simple model of the mounting process in order to predict performance. The prototype will be assembled at CPPI. The telescope will be evaluated for throughput and resolution using a pencil beam X-ray scanning system previously employed for evaluation of telescope prototypes ${ }^{11}$.

The first HXT prototype will have a $4.7 \mathrm{~m}$ focal length and the upper and lower layer lengths $15 \mathrm{~cm}$. Its $\sim 21 \mathrm{~cm}$ diameter is close to that utilized in our previous work on overconstrained mounting $(\sim 18 \mathrm{~cm})$. It will, however, have glass of comparable quality to that indicated in figure 1 . Thus it will be the first time the mounting process has been implemented with glass not limited by figure distortions.

A subsequent prototype will then be developed. This prototype will have to address additional issues related to the large range of radii spanned by the HXT. In particular the number of spacers to be employed per glass segment must be defined. Currently we use 3 or 5 such spacers per segment, with the larger number being used for larger radii. The conformance of the glass improves with increasing number of spacers as does the amount of obscuration of the X-ray beam. The stiffness of the glass sectors varies considerably from the inner radii to the outer radii, so there is the potential for varying levels of conformance of the glass via the overconstraint. In fact it is more difficult to constrain the stiffer inner layers and easier to constrain the outer layers. This is not likely to be an important effect for the eGlass since the overall glass figure is so good. In effect one expects that with the eGlass there will be little "constraint" at all, since there is no need to distort the glass from its natural shape other than the tiny amount necessary to bring it to the appropriate conic section. Nevertheless modeling efforts are underway to establish how many spacers will be appropriate for this prototype since its diameter of $40 \mathrm{~cm}$ is almost twice that of the initial HXT prototype. The current plan for this prototype is to have 4 shells around $20 \mathrm{~cm}$ diameter and $15 \mathrm{~cm}$ in segment length. This prototype will have eGlass mirrors with appropriate multilayer coatings so that it can be fully-characterized in X-ray tests. The cost and time required to procure mandrels makes it prohibitive to attempt to investigate mechanical issues associated with varying mirror radii with high quality replica mirrors. Consequently a second structural prototype will be built. This prototype will not have eGlass, but rather glass which is just formed on the mandrel. This glass will be have good enough figure quality to permit investigation of the mechanical issues involved in covering the large HXT radius range. This structural prototype will have shells at 10,20 and $40 \mathrm{~cm}$ diameter respectively.

\section{ACKNOWLEDGEMENTS}

This work is supported by a NASA grant to Columbia University: NAG5-5260 (Thermally Formed Optics for X-Ray and Gamma-Ray Astronomy), and by NASA grants to the California Institute of Technology with subawards to Columbia: Caltech No. 1019776 (High Energy Focussing Telescope) and Caltech No. 1046806 (Critical Technologies for theConstellation Hard X-Ray Telescope). 


\section{REFERENCES}

1. S.L. O'Dell, W.D. Jones, J.K. Russell, B.D. Ramsey, D.E. Engelhaupt, L.M. Cohen and L.P. Van Speybroeck, "Development of Constellation-X optics technology at MSFC," Proc. SPIE, 4012, 316, 2000.

2. R. Petre, L.M. Cohen, D.A. Content, J.D. Hein, T. Saha, M. Schattenburg and W. Zhang, "Progress towards meeting the Constellation-X performance goals segmented X-ray mirrors," Proc. SPIE, 4138, 2000.

3. W.W. Zhang, K. Chan, D.A. Content, R. Petre, P.J. Serlemitsos, T.T. Saha and Soong, "Development of mirror segments for the Constellation-X observatory," SPIE 4851, \#58, 2002.

4. W.W. Craig, F.E. Christensen, T.A. Decker, C.J. Hailey, F.A. Harrison, R.M. Hill, M. Jimenez-Garate, P.H. Mao and S.M. Schindler, "Hard X-ray optics for the HEFT balloon-borne payload: prototype design and status," Proc. SPIE, 3445, 112, 1998.

5. W.W. Craig, C. Hailey, M. Jimenez-Garate, D. Windt, F. Harrison, P. Mao, F. Christensen and A. Hussaiin, Optics Express 7, 178, 2000.

6. P.J. Serlemitsos and Y. Soong, "Foil X-ray Mirrors," Ap. Sp. Sci., 239, 177, 1996.

7. H.W. Schnopper, "SODART telescopes on the Spectrum-X Gamma (SRG) and their complemnent of instruments," SPIE 2279, 412, 1994.

8. J. Koglin, F. Christensen, J. Chonko, W. Craig, T. Decker, M. Jimenez-Garate, C. Hailey, F. Harrison, C. Jensen, M. Sileo, D. Windt and H. Yu, "Development and production of hard X-ray multilayer optics for HEFT," SPIE 4851, \#67, 2002.

9. C.J. Hailey, S. Abdali, F.E. Christensen, W.W. Craig, T.A. Decker, F.A. Harrison and M.A. Jimenez-Garate, "Substrates and Mounting Techniques for the High-Energy Focusing Telescope (HEFT), " Proc. SPIE, 3114, 535, 1997

10. J.E. Koglin, H.C. Chen, F.E. Christensen, J. Chonko, W.W. Craig, T.R. Decker, M.A.Jimenez-Garate, C.J. Hailey, F.A. Harrison, C.P. Jensen, M. Sileo, D.L. Windt and H.Yu, "Development of Hard X-ray Multilayer Optics with sub-arcminute performance," Proc. SPIE 4851, \#74, 2002.

11. F.E. Christensen, B.P. Byrnak, A. Hornstrup, Z.S. Hua, M.J. Westergaard and H.W. Schnopper, "X-ray scattering measurements from thin foil X-ray mirrors," SPIE 1546, 53, 1991. 